\title{
Análise das internações hospitalares para procedimentos de cirurgias bariátricas financiadas pelo SUS em âmbito nacional
}

\author{
Analysis of hospital admissions for bariatric surgeries financed by Public Health \\ System in Brazil
}

Eduardo Botti Abbade ${ }^{1}$ (D)

\begin{abstract}
RESUMO
Modelo do estudo: Estudo descritivo transversal. Objetivo: O objetivo deste estudo foi analisar a evolução do quantitativo e valor aprovado no âmbito do SUS, no período de 2008 a 2018, associado aos procedimentos cirúrgicos de gastroplastia (cirurgia bariátrica) relacionados ao diagnóstico principal de obesidade por excesso de ingestão de calorias. Método: Foi conduzido estudo ecológico com levantamento de dados obtidos nos Sistemas de Informações Hospitalares e Ambulatoriais do SUS (SIH/SUS e SAI/SUS). Foram levantados dados mensais do quantitativo e valor aprovado referentes aos procedimentos selecionados associados às cirurgias bariátricas em âmbito nacional, compreendendo o período de janeiro de 2008 a julho de 2018. Resultados: Os resultados mostram um crescimento acentuado na quantidade de procedimentos de gastroplastia financiados pelo SUS. Em 2017 foram financiados 10.840 procedimentos de gastroplastia, totalizando cerca de $\mathrm{R} \$ 69$ milhões. Em julho de 2018 o valor médio do custo ao SUS do procedimento de gastroplastia foi estimado em $\mathrm{R} \$ 6.449,30$. Observou-se que o tempo médio de internação e a taxa média de óbitos referentes a procedimentos de gastroplastia apresentam considerável redução. Também é evidenciado que as mulheres representam cerca de $85 \%$ dos pacientes de procedimentos de gastroplastia financiadas pelo SUS. Conclusão: A epidemia de obesidade que aflige a população brasileira ocasiona aumento do quantitativo de gastroplastias financiadas pelo SUS, que ainda são consideradas insuficientes, o que exige que sejam delineadas políticas e iniciativas públicas em prol do combate à obesidade coletiva no Brasil.
\end{abstract}

Palavras-chave: Obesidade; Custos e Análise de Custo; Sistema Único de Saúde; Cirurgia Bariátrica; Saúde Pública.

\begin{abstract}
Study design: Cross-sectional descriptive study. Objective: The objective of this study was to analyze the evolution of the quantitative and value approved by the Brazilian Unified Health System (SUS), from 2008 to 2018 , regarding surgical procedures of gastroplasty (bariatric surgery) related to the main diagnosis of obesity due to excess of calories ingestion. Methods: An ecological study was conducted with data collection obtained from the Hospital and Ambulatory Information Systems of SUS (SIH/SUS and SAI/SUS). Monthly data of the quantitative and approved value were collected regarding the procedures selected associated with bariatric surgeries at the national scope, covering the period from January 2008 to July 2018. Results: The results show a sharp increase in the number of procedures of gastroplasty financed by SUS. In 2017, 10,840 gastroplasty procedures were financed with public resources totaling approximately R\$ 69 million. In July 2018, the average cost of the gastroplasty procedure for SUS was estimated in $R \$ 6,449.30$. It was observed that the average length of stay and the average rate of deaths related to gastroplasty procedures presented considerable reduction. It is also evidenced that women represent about $85 \%$ of the patients for bariatric surgeries financed by SUS. Conclusions: The obesogenic epidemic afflicting the Brazilian population strongly increases in the number of gastroplasties financed by SUS, which are still considered insufficient, requiring proper public policies and initiatives to fight the collective obesity in Brazil.
\end{abstract}

Keywords: Obesity; Costs and Cost Analysis; Brazilian Unified Health System; Bariatric Surgery; Public Health.

1. Professor adjunto. Departamento de Ciências Administrativas, Universidade Federal de Santa Maria (UFSM), Santa Maria (RS), Brasil.

$\triangle \quad$ Av. Roraima, 1000, Prédio 74C - Camobi. CEP 97105-900. Santa Maria RS, Brasil. eduardo.abbade@ufsm.br I edabbade@hotmail.com | Recebido: 17/01/19 | Aprovado: 26/04/2019 


\section{INTRODUÇÃO}

A obesidade é uma doença multifatorial, crônica e progressiva, que afeta parcelas alarmantes da população mundial, atingindo o patamar de pandemia ${ }^{1-4}$. De fato, segundo a Organização Mundial da Saúde, mais de 1,9 bilhões de adultos (pessoas com mais de 18 anos) estavam acima do peso (39\%) em 2014, e mais de 600 milhões eram obesos $(13 \%)^{5}$. No Brasil, o elevado nível de obesidade observado na população é também preocupante, uma vez que, segundo dados do Ministério da Saúde, em 2013 cerca de 30\% das crianças do país apresentavam diagnóstico de sobrepeso ou obesidade. Além disso, dados do VIGITEL 2017, amplo estudo de vigilância de fatores de risco e proteção para doenças crônicas por inquérito telefônico feito anualmente pelo Ministério da Saúde, revelou que cerca de 18,9\% da população estava diagnosticada como obesa e $54 \%$ estava com sobrepeso ${ }^{6}$.

A obesidade coletiva observada em diversas populações em todo o mundo tem graves implicações para a saúde pública. Evidências de estudos anteriores mostram que a prevalência de obesidade aumenta o risco de morbidade e mortalidade por diabetes e doença cardiovascular ${ }^{7-9}$, implicando em um aumento dos gastos necessários no âmbito da saúde pública.

Evidências de estudos anteriores sugerem que a obesidade e as doenças relacionadas à obesidade resultam em gastos excessivos com a saúde ${ }^{10-12}$, além de implicações associadas a perdas de produtividade dos indivíduos ${ }^{13,14}$. De fato, estudo anterior demonstrou que os custos médicos associados à obesidade chegam a US $\$ 209,7$ biIhões nos Estados Unidos e que a redução efetiva do peso da população poderia gerar redução de custo na ordem de US\$ 610 bilhões em 20 anos $^{15}$.

A obesidade pode levar à diabetes, doenças cardiovasculares e alguns tipos de canceres, sendo estas decorrências usualmente consideradas na avaliação dos custos da obesidade à saúde ${ }^{16-19}$. No entanto, poucos estudos se dedicam a avaliar o custo para a saúde pública da realização de procedimentos hospitalares voltados ao tratamento da obesidade, como os procedimentos de cirurgia bariátrica. O que se observa são estudos que objetivam analisar o custo efetividade de cirurgias bariátricas ou os ganhos com o aumento da realização de cirurgias bariátricas ${ }^{20-22}$, ou ainda estudos que busquem estimar os custos decorrentes de diagnósticos de doenças relacionadas à obesidade, como diabetes, doenças cardiovasculares e depressão ${ }^{15,16,19,23-25}$. No entanto, cirurgias desta natureza não são ações preventivas, e implicam em custos hospitalares que poderiam ser evitados através de ações de prevenção à obesidade. Com isso, o objetivo deste estudo foi analisar a evolução do quantitativo e valor aprovado no âmbito do SUS, no período de 2008 a 2018, associado aos procedimentos cirúrgicos de gastroplastia (cirurgia bariátrica) relacionados ao diagnóstico principal de obesidade por ingestão de calorias.

\section{MATERIAL E MÉTODO}

Foi conduzido um estudo ecológico com o propósito de analisar a evolução e tendência de realização de procedimentos relacionados a diagnósticos de obesidade em hospitais públicos do Brasil, considerando o período de janeiro de 2008 a julho de 2018. Os dados secundários analisados neste estudo foram obtidos no DATASUS (www.datasus.gov.br), por acesso ao Sistema de Informações Hospitalares do Sistema Único de Saúde (SIH/SUS) e do Sistema de Informações Ambulatoriais do Sistema Único de Saúde (SIA/SUS).

Foi obtida a listagem de procedimentos hospitalares e ambulatoriais associados ao CID principal E660 (Obesidade devida a excesso de calorias), à habilitação hospitalar 0202 (Unidade de alta complexidade ao paciente portador de obesidade grave) e habilitação hospitalar 0203 (Assistência em Alta Complexidade ao indivíduo com Obesidade) no SIGTAP (Sistema de Gerenciamento da Tabela de Procedimentos, Medicamentos e OPM do SUS). Os procedimentos selecionados para análise são apresentados na Tabela 1. Foram desconsiderados 2 procedimentos associados ao CID principal E660 - 03.01.05.001-5 (Acompanhamento e avaliação domiciliar de paciente submetido à ventilação) e 03.02.04.002-1 (Atendimento fisioterapêutico em paciente com transtorno respiratório) por não serem procedimentos associados especificamente para procedimentos de cirurgia bariátrica. 


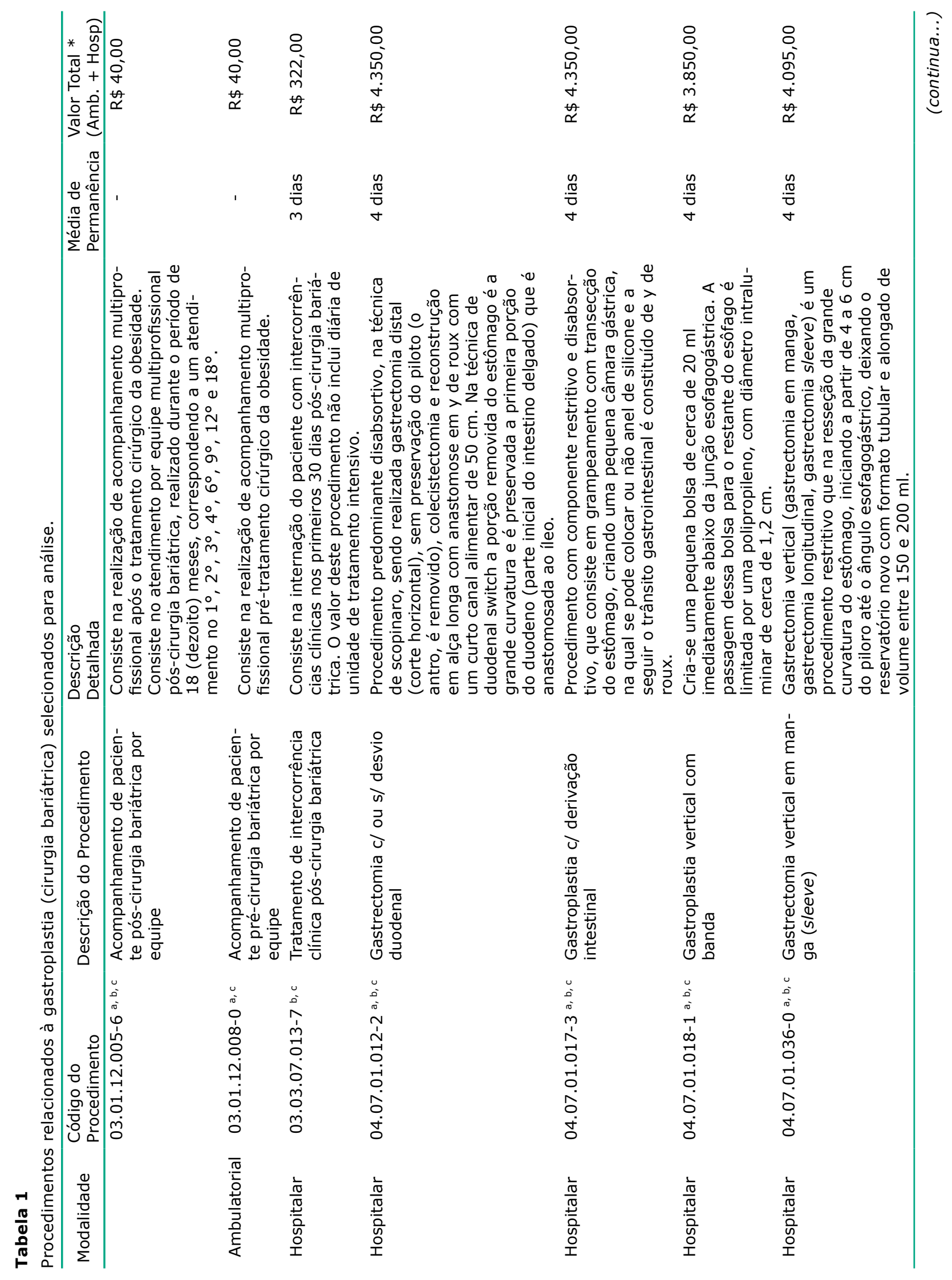




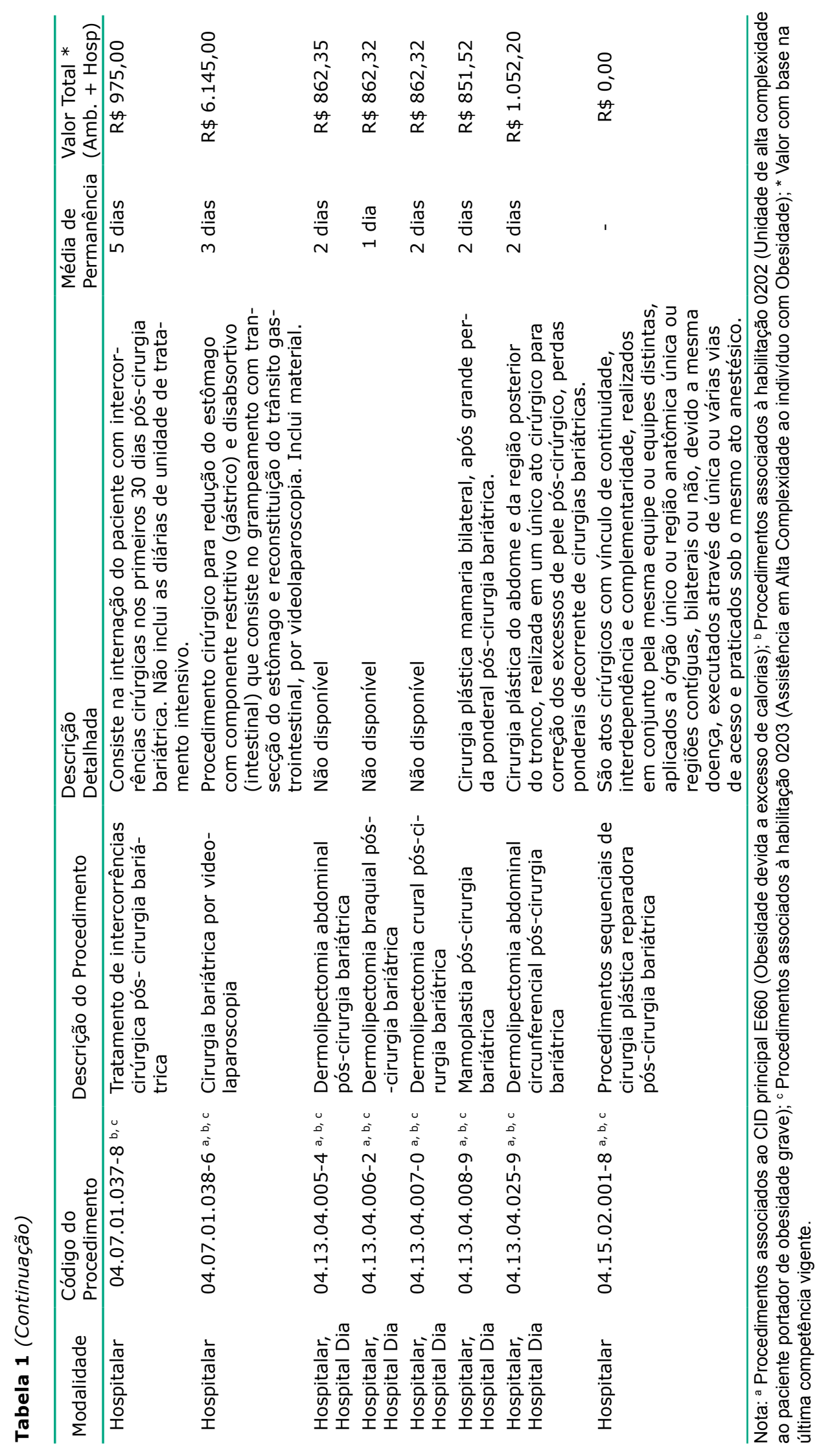


Para cada um dos procedimentos principais de internação hospitalar listados na Tabela 1, foram obtidos os valores totais mensais aprovados no período de jan./2008 a jul./2018 de todos os procedimentos secundários realizados, totalizando 210 procedimentos distintos. A contagem de procedimentos (principal + secundários) distintos considerados em cada um dos procedimentos elencados na Tabela 1 é apresentada na Tabela 2.

Também foram obtidos os quantitativos aprovados dos seguintes cinco procedimentos cirúrgicos principais, sendo estes considerados como Procedimentos Principais Maiores (PPMs):
(1) 04.07.01.012-2 [Gastrectomia c/ ou s/ desvio duodenal]; (2) 04.07.01.017-3 [Gastroplastia c/ derivação intestinal]; (3) 04.07.01.018-1 [Gastroplastia vertical com banda]; (4) 04.07.01.0360 [Gastrectomia vertical em manga (sleeve)]; e (5) 04.07.01.038-6 [Cirurgia bariátrica por videolaparoscopia]. A seleção destes cinco procedimentos se deu pelo fato de que os demais procedimentos selecionados para o estudo são procedimentos realizados antes ou após o Procedimento Principal Maior selecionado. Dessa forma, foi possível calcular o valor aprovado médio para cada PPM.

\section{Tabela 2}

Quantidade de Procedimentos distintos (principal + secundários) realizados sob o procedimento principal analisado

\begin{tabular}{lc}
\multicolumn{1}{c}{ Procedimento Principal } & $\begin{array}{c}\text { Contagem de Procedimentos } \\
\text { (principal + secundários) }\end{array}$ \\
\hline 03.01.12.005-6 - Acompanhamento de paciente pós-cirurgia bariátrica por equipe & 1 \\
03.01.12.008-0 - Acompanhamento de paciente pré-cirurgia bariátrica por equipe & 1 \\
03.03.07.013-7 - Tratamento de intercorrência clinica pós-cirurgia bariátrica & 95 \\
04.07.01.012-2 - Gastrectomia c/ ou s/ desvio duodenal & 74 \\
04.07.01.017-3 - Gastroplastia c/ derivação intestinal & 169 \\
04.07.01.018-1 - Gastroplastia vertical com banda & 74 \\
04.07.01.036-0 - Gastrectomia vertical em manga (sleeve) & 68 \\
04.07.01.037-8 - Tratamento de intercorrências cirúrgica pós-cirurgia bariátrica & 65 \\
04.07.01.038-6 - Cirurgia bariátrica por videolaparoscopia & 41 \\
04.13.04.005-4 - Dermolipectomia abdominal pós-cirurgia bariátrica & 75 \\
04.13.04.006-2 - Dermolipectomia braquial pós-cirurgia bariátrica & 23 \\
04.13.04.007-0 - Dermolipectomia crural pós-cirurgia bariátrica & 30 \\
04.13.04.008-9 - Mamoplastia pós-cirurgia bariátrica & 36 \\
04.13.04.025-9 - Dermolipectomia abdominal circunferencial pós-cirurgia bariátrica & 29 \\
04.15.02.001-8 - Procedimentos sequenciais de cirurgia plástica reparadora pós-cirurgia & 27 \\
bariátrica & 210 \\
\hline Contagem Total de Procedimentos Distintos & \\
\hline
\end{tabular}

Este estudo também analisou os dados anuais de estimativa de IMC médio da população do Brasil, compreendendo o período de 2008 a 2016, obtidos no repositório de dados da Organização Mundial da Saúde ${ }^{5}$. Os dados desta série temporal foram confrontados com os dados referentes à soma dos quantitativos anuais dos 5 PPMs relacionados às cirurgias bariátricas realizados no âmbito do SUS de modo a obter o grau de associação entre tais variáveis por meio do cálculo da Correlação de Pearson.

\section{RESULTADOS}

Ao analisar a evolução mensal, de janeiro de 2008 a julho de 2018, do total dos valores monetários aprovados de todos os procedimentos considerados neste estudo (principal + secundários), observa-se um acentuado crescimento. $O$ montante financeiro aprovado relacionado aos procedimentos investigados é apresentado na Figura 1. 
No mês de julho de 2018 foi registrado um valor total aprovado, considerando a soma dos valores de todos os procedimentos avaliados no estudo, de R\$ 6,5 milhões. No ano de 2008, o valor total aprovado pelo SUS referente aos procedimentos analisados no estudo foi de $R \$$ 16.218.343,93. Já em 2017, o valor total aprovado pelo SUS foi de $\mathrm{R} \$ 69.250 .839,51$, repre- sentando um crescimento de cerca de $327 \%$. Os resultados também sugerem uma tendência de crescimento médio no valor de $R \$ 40.041,00$ por mês, estimada por uma regressão linear simples. Na Figura 1, destaque é dado para o procedimento principal 04.07.01.017-3 (área azul do gráfico) por ser o procedimento com maior representatividade em termos de valor monetário aprovado.

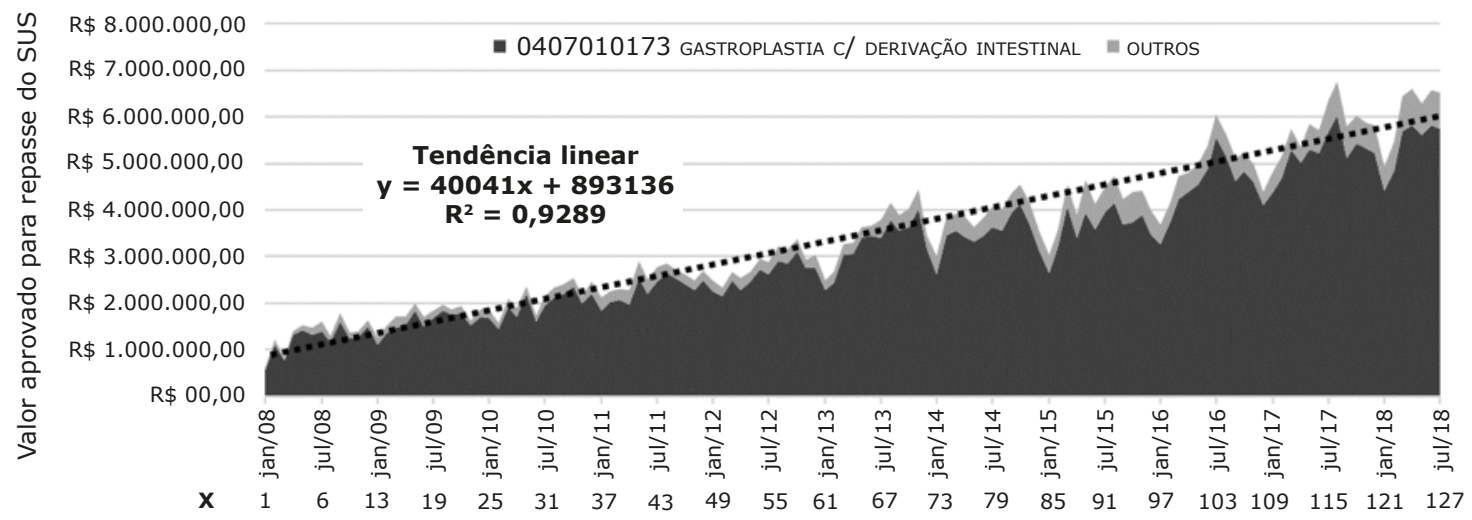

Figura 1: Evolução mensal do montante financeiro aprovado referente aos procedimentos relacionados a procedimentos de gastroplastia (principais e secundários)

\section{Tabela 3}

Evolução anual da quantidade dos 5 Procedimentos Principais Maiores, somatório do valor aprovado pelo SUS para os 5 Procedimentos Principais Maiores e valor médio por Procedimento Principal Maior aprovado.

\begin{tabular}{ccccccc}
\hline ANO & $\begin{array}{c}\text { IMC médio } \\
\text { populacional }\end{array}$ & $\begin{array}{c}\text { Qtd. } \\
\text { PPMs* }\end{array}$ & $\begin{array}{c}\text { Soma do Valor } \\
\text { de todos os } \\
\text { procedimentos } \\
\text { relacionados }\end{array}$ & $\begin{array}{c}\text { Valor Médio por } \\
\text { Paciente submetido } \\
\text { a um dos PPMs* }\end{array}$ & $\begin{array}{c}\text { Tempo } \\
\text { Médio de } \\
\text { Permanência } \\
\text { dos PPMs* }\end{array}$ & $\begin{array}{c}\text { Taxa média } \\
\text { de óbito nos } \\
\text { PPMs }\end{array}$ \\
\hline 2008 & 25,6 & 3258 & $\mathrm{R} \$ 16.218 .343,93$ & $\mathrm{R} \$ 4.978,01$ & 5,7 & $0,64 \%$ \\
2009 & 25,7 & 3923 & $\mathrm{R} \$ 21.099 .291,63$ & $\mathrm{R} \$ 5.378,36$ & 5,3 & $0,45 \%$ \\
2010 & 25,8 & 4815 & $\mathrm{R} \$ 25.588 .180,20$ & $\mathrm{R} \$ 5.314,26$ & 5 & $0,46 \%$ \\
2011 & 26 & 5809 & $\mathrm{R} \$ 30.652 .124,64$ & $\mathrm{R} \$ 5.276,66$ & 4,5 & $0,31 \%$ \\
2012 & 26,1 & 6432 & $\mathrm{R} \$ 34.260 .257,41$ & $\mathrm{R} \$ 5.326,53$ & 4,4 & $0,40 \%$ \\
2013 & 26,2 & 7257 & $\mathrm{R} \$ 42.811 .406,62$ & $\mathrm{R} \$ 5.899,33$ & 4,1 & $0,28 \%$ \\
2014 & 26,3 & 7568 & $\mathrm{R} \$ 46.815 .102,96$ & $\mathrm{R} \$ 6.185,93$ & 4 & $0,27 \%$ \\
2015 & 26,5 & 8024 & $\mathrm{R} \$ 50.145 .488,99$ & $\mathrm{R} \$ 6.249,44$ & 3,9 & $0,20 \%$ \\
2016 & 26,6 & 9393 & $\mathrm{R} \$ 59.191 .162,10$ & $\mathrm{R} \$ 6.301,62$ & 3,5 & $0,33 \%$ \\
2017 & $\mathrm{ND}$ & 10840 & $\mathrm{R} \$ 69.250 .839,51$ & $\mathrm{R} \$ 6.388,45$ & 3,1 & $0,22 \%$ \\
$2018 * *$ & $\mathrm{ND}$ & 6644 & $\mathrm{R} \$ 42.849 .168,09$ & $\mathrm{R} \$ 6.449,30$ & 2,9 & $0,19 \%$ \\
\hline
\end{tabular}

Nota: ND = Não Disponível; PPMs são os 5 procedimentos cirúrgicos principais de gastroplastia considerados no estudo (04.07.01.012-2 [Gastrectomia c/ ou s/ desvio duodenal], 04.07.01.017-3 [Gastroplastia c/ derivação intestinal], 04.07.01.018-1 [Gastroplastia vertical com banda], 04.07.01.036-0 [Gastrectomia vertical em manga (sleeve)] e 04.07.01.038-6 [Cirurgia bariátrica por videolaparoscopia]); ** dados até Julho de 2018. 
Ao analisar o quantitativo anual aprovado pelo SUS, dos cinco Procedimentos Principais Maiores considerados neste estudo, efetuou-se o cálculo do valor médio anual do procedimento cirúrgico bariátrico. Os resultados de tal análise são apresentados na Tabela 3.

Os resultados apresentados na Tabela 3 sugerem que a quantidade anual dos Procedimentos de Gastroplastia feitos e aprovados pelo SUS apresentam crescimento, ao passo que em 2008 foram aprovados 3.258 procedimentos do grupo dos cinco procedimentos principais considerados, e em 2017 foram aprovados 10.840 procedimentos do mesmo grupo. Ademais, o valor médio do procedimento de gastroplastia aumentou de $\mathrm{R} \$ 4.978,01$ em 2008 para $\mathrm{R} \$ 6.449,30$ em 2018 (considerando dados até julho). Tal evidência sugere um crescimento acentuado no valor aprovado para repasse pelo SUS para os hospitais no que se refere a cada paciente submetido ao procedimento de gastroplastia. Logo, é possível sugerir que o custo de um paciente submetido ao procedimento de gastroplastia feito pelo SUS possui um custo médio de $\mathrm{R} \$ 6.449,30$. Ademais, esta estimativa de custo não incorpora elementos importantes como o risco de óbito, permanência de internação e ocupação de leitos hospitalares e eventuais complicações futuras em virtude do procedimento cirúrgico.

Estudo conduzido na Europa estimou que o custo imediato de 1.000 procedimentos cirúrgicos bariátricos seria de cerca de $£ 9,16$ milhões, com custos de cuidados de saúde incrementais de $£ 15,26$ milhões $^{26}$. Outro estudo relevante conduzido na Espanha evidenciou que ao longo de um horizonte temporal de 10 anos, a cirurgia bariátrica apresenta um incremento de custo de $€ 9.386$ e um ganho de 1,6 QALY (quality-adjusted life years). Além disso, o estudo evidenciou que a cirurgia bariátrica teria potencial de reduzir significativamente o risco de diabetes e doenças cardiovasculares ao longo dos horizontes considerados, apresentando ganho de 0,6 LYG (life years gained) e 4,4 QALY, além de economias de custo médio de $€ 300 /$ paciente ao longo da vida ${ }^{27}$.

Outro ponto que merece destaque neste estudo são os resultados obtidos que sugerem que o tempo médio de internação e a taxa média de óbitos referentes aos procedimentos de cirurgia bariátrica estão diminuindo, o que é considerado um resultado altamente positivo para o quadro da saúde no Brasil. De fato, em 2008, o tempo médio de permanência de pacientes internados para a realização de procedimentos de cirurgia bariátrica era de 5,7 dias, ao passo que em 2018 o tempo médio registrado foi de 2,9 dias. Já a taxa de óbito em 2008 registrada foi de $0,64 \%$, sendo que em 2018 a taxa média de óbito registrada foi de $0,19 \%$. Tais resultados sugerem uma melhoria na qualidade da internação hospitalar referente aos procedimentos de cirurgias bariátricas.

Inúmeros estudos corroboram a premissa de que a cirurgia bariátrica é custo-efetiva ${ }^{20,22,28,29}$. No entanto, é necessário destacar que a cirurgia bariátrica é intervenção de risco. Logo, é necessário considerar intervenções em âmbito social e ambiental que auxiliem na promoção da redução do nível de obesidade populacional e que possam combater a consolidação de ambientes obesogênicos ${ }^{1,4}$.

De modo a complementar a análise da evolução do quantitativo de procedimentos de gastroplastia realizados e aprovados pelo SUS em âmbito nacional, efetuou-se o cálculo da correlação do quantitativo dos 5 Procedimentos Principais Maiores com a evolução do IMC médio da população brasileira. Os resultados mostram um crescimento acentuado no quantitativo de procedimentos de gastroplastia (os 5 PPMs do estudo) realizados pelo SUS anualmente no Brasil. Em 2008 foram registrados 3.258 procedimentos realizados e em 2017 o total de procedimentos realizados e aprovados pelo SUS foi de cerca de 10.840 , indicando um crescimento de $232,72 \%$ no período de nove anos. Além disso, os resultados também sugerem a existência de uma forte correlação positiva e significativa entre o quantitativo de procedimentos de gastroplastia aprovados e o IMC médio da população Brasileira ( $\rho=0,9901 ; p<0,0001)$, que também apresenta acentuado crescimento.

O perfil dos pacientes cujos 5 PPMs relacionados à gastroplastia considerados neste estudo que foram aprovados pelo SUS no período de janeiro de 2017 a julho de 2018 é apresentado na Tabela 4.

A análise dos dados disponibilizados pelo SIH/SUS revela que a idade média dos pacientes que são submetidos a procedimentos de gastroplastia é de cerca de 40 anos. Os dados também mostram que as mulheres representam cerca de $85 \%$ dos pacientes que realizam procedimentos 
de gastroplastia via SUS. Os dados da Organização Mundial da Saúde revelam que o IMC médio das mulheres é usualmente superior ao dos homens, tanto no Brasil quanto na média mundial ${ }^{5}$. Ademais, a obesidade em mulheres apresenta implicações distintas ao considerar que a obesidade apresenta maior prevalência entre mulheres do que homens e que a obesidade entre mulheres em condições reprodutivas pode estar associada à obesidade infantil ${ }^{30}$. De fato, estudos sugerem que mulheres em idade reprodutiva estão mais susceptíveis ao ganho de peso e à obesidade ${ }^{31}$, sendo que tal ganho de peso comumente ocorre durante a gravidez ou logo após o parto ${ }^{32,33}$. Com isso, a obesidade de mulheres em idade reprodutiva representa problemática urgente à saúde pública, já que pode trazer implicações severas tanto para a mulher como para a criança.

\section{Tabela 4}

Quantidade de procedimentos realizados aprovados, idade média e sexo dos pacientes dos 5 PPMs.

\begin{tabular}{|c|c|c|c|c|}
\hline & \multirow{2}{*}{ Qtd. } & \multirow{2}{*}{$\begin{array}{c}\text { Idade } \\
\text { (média } \pm \text { desvio } \\
\text { padrão) }\end{array}$} & \multicolumn{2}{|c|}{ Sexo } \\
\hline & & & Homens & Mulheres \\
\hline 04.07.01.012-2 [Gastrectomia c/ ou s/ desvio duodenal] & 29 & $52,38 \pm 15,54$ & $\begin{array}{c}12 \\
(41,38 \%)\end{array}$ & $\begin{array}{c}17 \\
(58,62 \%)\end{array}$ \\
\hline 04.07.01.017-3 [Gastroplastia c/ derivação intestinal] & 15.731 & $39,16 \pm 10,63$ & $\begin{array}{c}2.333 \\
(14,83 \%)\end{array}$ & $\begin{array}{c}13.398 \\
(85,17 \%)\end{array}$ \\
\hline 04.07.01.018-1 [Gastroplastia vertical com banda] & 65 & $42,38 \pm 11,64$ & $\begin{array}{c}13 \\
(20 \%)\end{array}$ & $\begin{array}{c}52 \\
(80 \%)\end{array}$ \\
\hline 04.07.01.036-0 [Gastrectomia vertical em manga (sleeve)] & 340 & $40,89 \pm 11,12$ & $\begin{array}{c}85 \\
(25 \%)\end{array}$ & $\begin{array}{c}255 \\
(75 \%)\end{array}$ \\
\hline 04.07.01.038-6 [Cirurgia bariátrica por videolaparoscopia] & 503 & $40,22 \pm 10,19$ & $\begin{array}{c}71 \\
(14,12 \%)\end{array}$ & $\begin{array}{c}432 \\
(85,88 \%)\end{array}$ \\
\hline Total & 16.668 & $39,26 \pm 10,66$ & $\begin{array}{c}2.514 \\
(15,08 \%)\end{array}$ & $\begin{array}{c}14.154 \\
(84,92 \%)\end{array}$ \\
\hline
\end{tabular}

\section{DISCUSSÃO}

O Brasil é o segundo país do mundo em termos de quantidade de procedimentos cirúrgicos bariátricos realizados anualmente, ficando atrás apenas dos Estados Unidos, segundo dados da International Federation for the Surgery of Obesity and Metabolic Disorders (IFSO). Dados da pesquisa do IFSO de 2016 revelaram que foram realizados cerca de 114 mil procedimentos bariátricos/metabólicos no Brasil ${ }^{34}$. Evidência de estudos anteriores mostram que, apesar do aumento do quantitativo de cirurgias bariátricas realizadas na rede pública de saúde brasileira, este número ainda é considerado insuficiente ${ }^{35}$. De fato, a rede pública de saúde do Brasil realiza (ou financia/ aprova) cerca de apenas $8 \%$ dos procedimentos de cirurgias bariátricas de sua população, já que em 2016 foram aprovadas 9.393 cirurgias bariátricas pelo SUS (Tabela 3). Portanto, a oferta de cirurgias bariátricas via SUS no Brasil, apesar de crescente, ainda é considerada insuficiente.
A cirurgia bariátrica, desde que realizada de forma adequada, é considerada método seguro e eficaz para o tratamento da obesidade e comorbidades, proporcionando potencial redução de custos em saúde ${ }^{36}$. Ademais, se por um lado a gastroplastia pode ser considerada intervenção de risco, por outro, há estudos que demonstram que a precocidade da indicação da cirurgia bariátrica está associada a melhores resultados, especialmente em relação a diabetes e doença renal ${ }^{37,38}$. Dessa forma, delineamentos avançados de propostas de ampliação da oferta de cirurgias bariátricas no âmbito do SUS, juntamente com definição de protocolos médicos mais eficientes em termos de custo-efetividade se fazem necessários.

Além de iniciativas direcionadas ao sistema público de saúde, promover um melhor entendimento das causas ambientais e sociais que fomentam a obesidade coletiva aos gestores públicos, e suas implicações para a sociedade em termos sociais, econômicos e ambientais, também se faz necessário. A exemplo disso, estudo reve- 
la que o nível de desemprego está positivamente associado ao quantitativo de cirurgias bariátricas realizadas em âmbito populacional via rede pública de saúde do Brasil ${ }^{35}$. Por outro lado, estudos também revelam que populações obesas tendem a apresentar menores índices de produtividade no trabalho ${ }^{39,40}$. Logo, combater a obesidade coletiva com métodos eficientes de tratamentos ofertados pelo sistema de saúde pública podem promover reduções significativas no custo associado a outras morbidades e promover aumento no desempenho produtivo dos indivíduos.

Algumas limitações merecem destaque ao passo que este estudo não avalia o custo-efetividade dos procedimentos de cirurgia bariátrica realizados pelo SUS, não avalia as eventuais economias geradas em virtude de tais procedimentos considerando os riscos de doenças cardíacas, diabetes e outras enfermidades que pacientes obesos possam vir a apresentar, e não avalia os ganhos psicológicos e sociais que os indivíduos submetidos aos procedimentos de cirurgia bariátrica possam vir a apresentar, como por exemplo, melhoria em sua condição social e produtividade no trabaIho. $O$ que este estudo mostra é que os custos para - SUS relacionados aos procedimentos de cirurgia bariátrica estão aumentando, o que demanda que sejam delineadas políticas públicas para o combate à obesidade coletiva que se agrava no Brasil.

Como sugestão de estudos futuros, seria importante analisar profundamente o perfil dos pacientes que são submetidos aos procedimentos de cirurgia bariátrica pelo SUS, identificando aspectos como eventuais diagnósticos de transtornos e enfermidades psicológicas, hábitos alimentares, etc. Outra oportunidade de pesquisa é a análise dos quantitativos realizados e aprovados pelo SUS por região geográfica do Brasil. Uma análise mais detalhada das bases de dados do SUS apresenta potencial importante para meIhor elucidar a questão abordada neste estudo.

\section{CONCLUSÃO}

Este estudo teve como propósito avaliar o custo geral para o SUS em termos de aprovação e repasse às instituições hospitalares gerado pelos procedimentos de cirurgias bariátricas realizados e aprovados em âmbito nacional. Fica evidenciado que o custo para o SUS no que se refere aos procedimentos de cirurgia bariátrica e procedimentos secundários relacionados é crescente e em montante significativo. De fato, a problemática da obesidade coletiva que aflige a população brasileira está associada ao aumento significativo da quantidade de procedimentos de cirurgias bariátricas realizadas no país. Tais constatações reforçam a necessidade de serem delineadas políticas e iniciativas públicas em prol do combate à obesidade coletiva no Brasil e de melhor ajustamento da oferta de cirurgias bariátricas via SUS à população.

Apesar de estudos anteriores apresentarem inúmeros argumentos em favor da realização de cirurgias bariátricas como meio de combater a obesidade e promover melhorias à saúde coletiva, é importante argumentar em favor da promoção de iniciativas públicas com foco na prevenção da obesidade coletiva, combatendo as causas ambientais e comportamentais que agravam a obesidade ao nível populacional. Logo, políticas públicas que tenham como propósito promover a redução dos elevados níveis de obesidade e sobrepeso nas populações adulta e infantil do Brasil são altamente necessárias e encorajadas. Ademais, é importante que tais iniciativas tenham forte embasamento em evidências científicas de modo a alcançarem maior efetividade.

\section{REFERÊNCIAS}

1. Abbade EB, Dewes H. Behavioral and societal drivers of an obesogenic environment worldwide. Nutr Food Sci. 2015;45:229-241.

2. Giskes K, van Lenthe F, Avendano-Pabon M, Brug J. A systematic review of environmental factors and obesogenic dietary intakes among adults: Are we getting closer to understanding obesogenic environments? Obes Rev. 2011;12(5):e95-e106.

3. Prentice AM. The emerging epidemic of obesity in developing countries. Int J Epidemiol. 2006; 35(1):93-99.

4. Swinburn B, Egger G, Raza F. Dissecting obesogenic environments: The development and application of a framework for identifying and prioritizing environmental interventions for obesity. Prev Med. 1999;29:563-570.

5. OMS [Organização Mundial da Saúde]. Global Health Observatory (GHO), http://www.who.int/gho/database/ en/ (2018, accessed 5 August 2018). 
6. MS [Ministério da Saúde]. Vigilância de Fatores de Risco e Proteção para Doenças Crônicas por Inquérito Telefônico, Vigitel 2017. Ministério da Saúde Brasília, http://bvsms.saude.gov.br/bvs/publicacoes/vigitel_brasil_2017_vigilancia_fatores_riscos.pdf (2017).

7. McTigue K, Larson JC, Valoski A, Burke G, Kotchen J, Lewis $C E$, et al. Mortality and cardiac and vascular outcomes in extremely obese women. J Am Med Assoc. $2006 ; 296(1): 79-86$.

8. Misra A, Khurana L. Obesity and the metabolic syndrome in developing countries. J Clin Endocrinol Metab. 2008;93(11 Suppl 1):s9-30.

9. Moghaddam AA, Woodward M, Huxley R. Obesity and risk of colorectal cancer: a meta-analysis of 31 studies with 70,000 events. Cancer Epidemiol Biomarkers Prev. $2007 ; 16(12): 2533-47$

10. Abbade EB. The relationships between obesity-increasing risk factors for public health, environmental impacts, and health expenditures worldwide. Manag Environ Qual Int J. 2018;29(1):131-47.

11. Buchmueller TC, Johar M. Obesity and health expenditures: Evidence from Australia. Econ Hum Biol. 2015; 17:42-58.

12. Specchia ML, Veneziano MA, Cadeddu C, Ferriero AM, Mancuso $A$, Ianuale $C$, et al. Economic impact of adult obesity on health systems: a systematic review. Eur ] Public Health. 2015;25(2):255-262.

13. Adams KT. Managing the high cost of obesity. Manag Care Langhorne Pa 2015; 24: 42-44.

14. Colditz GA. Economic costs of obesity and inactivity. Med Sci Sports Exerc. 1999;31(11 Suppl):S663-7.

15. Spieker EA, Pyzocha N. Economic Impact of Obesity. Prim Care. 2016;43(1):83-95.

16. Buehler L, Fayfman M, Alexopoulos AS, Zhao L, Farrokhi $F$, Weaver J, et al. The impact of hyperglycemia and obesity on hospitalization costs and clinical outcome in general surgery patients. J Diabetes Complications. $2015 ; 29(8): 1177-82$.

17. Clarke PM, Glasziou P, Patel A, Chalmers J, Woodward M, Harrap SB, et al. Event rates, hospital utilization, and costs associated with major complications of diabetes: a multicountry comparative analysis. PLoS Med. 2010;7(2). doi: 10.1371/journal.pmed.1000236.

18. Nichols GA, Brown JB. The impact of cardiovascular disease on medical care costs in subjects with and without type 2 diabetes. Diabetes Care. 2002;25(3):482-6.

19. Simon GE, Katon WJ, Lin EH, Ludman E, VonKorff $M$, Ciechanowski $\mathrm{P}$, et al. Diabetes complications and depression as predictors of health service costs. Gen Hosp Psychiatry. 2005;27(5):344-51.

20. Alsumali A, Eguale T, Bairdain S, Samnaliev M. Cost-Effectiveness Analysis of Bariatric Surgery for Morbid Obesity. Obes Surg. 2018;28(8):2203-14.

21. Doble B, Wordsworth S, Rogers CA, Welbourn R, Byrne J, Blazeby JM, et al. What Are the Real Procedu- ral Costs of Bariatric Surgery? A Systematic Literature Review of Published Cost Analyses. Obes Surg. 2017;27(8):2179-92.

22. James R, Salton RI, Byrnes JM, Scuffham PA. Cost-utility analysis for bariatric surgery compared with usual care for the treatment of obesity in Australia. Surg Obes Relat Dis. 2017;13(12):2012-20.

23. Bueno DR, Marucci MFN, Gouveia LA, Duarte YAO, Lebrão ML. Abdominal obesity and healthcare costs related to hypertension and diabetes in older adults. Rev Nutr. 2017;30(2):209-18.

24. Egede LE, Zheng D, Simpson K. Comorbid depression is associated with increased health care use and expenditures in individuals with diabetes. Diabetes Care. 2002;25(3):464-70.

25. Finkelstein EA, Bray JW, Chen $\mathrm{H}$, Larson MJ, Miller $\mathrm{K}$, Tompkins $\mathrm{C}$, et al. Prevalence and costs of major depression among elderly claimants with diabetes. Diabetes Care. 2003;26(2):415-20.

26. Gulliford MC, Charlton J, Prevost T, Booth H, Fildes A, Ashworth M, et al. Costs and Outcomes of Increasing Access to Bariatric Surgery: Cohort Study and Cost-Effectiveness Analysis Using Electronic Health Records. Value Health. 2017;20(1):85-92.

27. Sanchez-Santos R, Padin EM, Adam D, Borisenko O, Fernandez SE, Dacosta EC, et al. Bariatric surgery versus conservative management for morbidly obese patients in Spain: a cost-effectiveness analysis. Expert Rev Pharmacoecon Outcomes Res. 2018;18(3):305-14.

28. Borisenko O, Lukyanov V, Debergh I, Dillemans B. Cost-effectiveness analysis of bariatric surgery for morbid obesity in Belgium. J Med Econ. 2018; 21(4):365-73.

29. Wentworth JM, Dalziel KM, O'Brien PE, Burton P, Shaba $F$, Clarke PM, et al. Cost-effectiveness of gastric band surgery for overweight but not obese adults with type 2 diabetes in the U.S. J Diabetes Complications. 2017;31(7):1139-44.

30. Tauqeer Z, Gomez G, Stanford FC. Obesity in Women: Insights for the Clinician. J Womens Health $2018 ; 27(4): 444-57$.

31. Harrison $\mathrm{CL}$, Skouteris $\mathrm{H}$, Boyle J, Teede HJ. Preventing obesity across the preconception, pregnancy and postpartum cycle: Implementing research into practice. Midwifery. 2017;52:64-70.

32. Fraser A, Tilling $K$, Macdonald-Wallis $C$, Hughes $R$, Sattar N, Nelson SM, et al. Associations of gestational weight gain with maternal body mass index, waist circumference, and blood pressure measured 16 y after pregnancy: the avon longitudinal study of parents and Children (ALSPAC). Am J Clin Nutr. 2011;93(6):1285-92.

33. Johnson J, Clifton RG, Roberts JM, Myatt L, Hauth JC, Spong $\mathrm{CY}$, et al. Pregnancy outcomes with weight gain above or below the 2009 institute of medicine guidelines. Obstet Gynecol. 2013;121(5):969-75.

34. Angrisani L, Santonicola A, Iovino P, Vitiello A, Higa K, Himpens J, et al. IFSO Worldwide Survey 2016: Primary, 
Endoluminal, and Revisional Procedures. Obes Surg. 2018;28(12):3783-94.

35. Cazzo E, Ramos AC, Chaim EA. Bariatric Surgery Offer in Brazil: a Macroeconomic Analysis of the Health system's Inequalities. Obes Surg. 2019;29(6):1874-80. doi: 10.1007/s11695-019-03761-3.

36. Borisenko $O$, Adam $D$, Funch-Jensen $P$, Ahmed $A R$, Zhang R, Colpan Z, et al. Bariatric Surgery can Lead to Net Cost Savings to Health Care Systems: Results from a Comprehensive European Decision Analytic Model. Obes Surg. 2015;25(9):1559-68.

37. Chang AR, Chen $Y$, Still $C$, Wood GC, Kirchner $H L$, Lewis $M$, et al. Bariatric surgery is associated with improvement in kidney outcomes. Kidney Int. 2016;90(1):164-171.

38. Jamal $M H$, Corcelles $R$, Daigle $C R$, Rogula $T$, Kroh $M$, Schauer PR, et al. Safety and effectiveness of bariatric surgery in dialysis patients and kidney transplantation candidates. Surg Obes Relat Dis. 2015;11(2):419-23.

39. Goettler A, Grosse A, Sonntag D. Productivity loss due to overweight and obesity: A systematic review of indirect costs. BMJ Open. 2017;7. doi:10.1136/bmjopen-2016-014632.

40. Kudel I, Huang JC, Ganguly R. Impact of Obesity on Work Productivity in Different US Occupations: Analysis of the National Health and Wellness Survey 2014 to 2015. J Occup Environ Med. 2018;60(1):6-11. 